\title{
Superintegrable systems
}

\author{
(supersymmetry/Lax equations/conservation laws)
}

\section{BORIS KUPERSHMIDT}

The University of Tennessee Space Institute, Tullahoma, TN 37388; and Center for Nonlinear Studies, Los Alamos National Laboratory, Los Alamos, NM 87545

Communicated by Peter D. Lax, June 15, 1984

\begin{abstract}
Families of matrix differential superintegrable systems of Lax type are constructed. Each family is a commutative Lie superalgebra with an infinite common set of conservation laws.
\end{abstract}

Introduction. Since the recent discovery of integrability of the Korteweg-de Vries equation, there has been a rapid growth of what is nowadays called the theory of integrable systems. This theory, still in active development, has acquired by now a clear mathematical foundation (1) and a number of deep results $(2,3)$. Meanwhile, the physical part of the theory-i.e., the study of particle-like behavior of nonlinear classical fields (4)-has branched out into a new and exciting area of "supersymmetry" (see, e.g., refs. 5 and 6), where anticommutative variables of the Grassmann type are treated on an equal footing with commutative variables of the usual mathematics. This development has influenced an initial opening in the area that, for want of a better name, I shall call superintegrable systems. Among them are supersymmetric two-dimensional $\sigma$-models (7) and supersymmetric two-dimensional Toda lattices $(8)$ associated with classical Lie superalgebras (9). Although none of these systems are of evolution type, one knows from the commutative theory that the Toda-type equations are intimately connected with the modified Lax equations, which are of evolution type $(2,3,10)$. This suggests that evolutionary superintegrable families exist, and I show below that this is indeed the case. The plan is as follows. In the first section we adjust the basic notions of commutative differential algebra to incorporate anticommuting variables. In the second section I describe the structure of the centralizer of a nondegenerate even semisimple matrix differential operator. In the next section the Lax equations are introduced and their basic properties are stated. In the last section a construction is given of an infinite set of common conservation laws for each commuting set of Lax equations, variational derivatives of these conservation laws are computed, and the nonHamiltonian character of the theory is explained. Superintegrable systems connected with the basic classical simple Lie superalgebras are left out of the paper; as is clear from ref. 3, for their construction one needs only to use the basic structural facts of the theory (which are given in Sections 2-4 below) together with the properties of the root space decomposition (which are given in ref. 9). I have assumed known, and tried to stay as close as possible to, the basic constructions of the commutative theory of ref. 1 . The ideas of ref. 11 for the residue calculus of pseudodifferential operators in the form given in ref. 12 are adapted in supersymmetric setting in Section 4. Finally, a few elementary facts about superalgebras and modules over them can be found in refs. 9 and 13 .

1. The Objects. Let $k^{\prime}$ be a commutative Q-algebra with unity, $k$ a commutative superalgebra over $k^{\prime}$, also with unity, $C$ a commutative superalgebra and a $k$-bimodule, $C^{\prime}$ an asso-

The publication costs of this article were defrayed in part by page charge payment. This article must therefore be hereby marked "advertisement" in accordance with 18 U.S.C. $\$ 1734$ solely to indicate this fact. ciative superalgebra and a $C$-bimodule, $\Omega$ a $\mathbf{Z}_{2}$-graded $C$-bimodule, and $\Omega^{\prime}$ a $Z_{2}$-graded $C^{\prime}$-bimodule. We denote $p:(\cdot)$ $\rightarrow \mathbf{Z}_{2}$ the parity of $\mathbf{Z}_{2}$-homogeneous elements in superalgebras and modules over them. These homogeneous elements are called $p$-homogeneous. All formulae written for $p$-homogeneous elements are extended by additivity to all elements.

We fix two nonnegative integers $\ell_{0}$ and $\ell_{1}$, at least one of which is positive, set $\ell=\ell_{0}+\ell_{1}$, and for a $Z_{2}$-graded vector space $V$ denote by $\bigcirc_{V}$ the supermatrix $\left(\ell_{0}, \ell_{1}\right)$-structure consisting of $\ell \times \ell$ matrices with entries in $V$ and the grading $p$ on $\bigcirc_{V}$ given as $p(X)=p\left(X_{\alpha \beta}\right)+p(\alpha)+p(\beta), X \in \bigcirc_{V}$, where $p(\alpha)=0$ for $\alpha \leq \ell_{0}$ and $p(\alpha)=1$ for $\alpha>\ell_{0}$. In these notations, $O_{C^{\prime}}$ becomes an associative superalgebra and a $\bigcirc_{C^{-}}$ bimodule, and $\mathrm{O}_{\Omega^{\prime}}$, becomes a $\mathbf{Z}_{2}$-graded $\mathrm{O}_{C^{\prime}}$-bimodule.

If $C^{\prime \prime}$ is an associative superalgebra, we denote $\left(C^{\prime \prime}\right)^{\text {Lie }}$ the corresponding Lie superalgebra with the bracket $[a, b]=a b$ $-(-1)^{p(a) p(b)} b a$ (see ref. 9).

We now specialize $C=k\left[u_{i, \alpha \beta}^{(m)}\right], m \in \mathbf{Z}_{+}, 1 \leq \alpha, \beta \leq \ell$, where the range for $\{(i, \alpha \beta)\}$ will be specified later on. The grading $p$ on $C$ is defined as $p\left(u_{i, \alpha \beta}^{(m)}\right)=p(\alpha)+p(\beta)$. Let $\partial: C$ $\rightarrow C$ be an even derivation of $C$ over $k$ acting on generators of $C$ as $\partial\left(u_{i, \alpha \beta}^{(m)}\right)=u_{i, \alpha \beta}^{(m+1)}$ (all derivations in this paper are left derivations). Set $\Omega=\Omega^{1}(C)=\left\{\Sigma d u_{i, \alpha \beta}^{(m)} \varphi_{i, \alpha \beta}^{m} \mid \varphi_{i, \alpha \beta}^{m} \in C\right.$, finite sums $\}$, and let $d: C \rightarrow \Omega^{1}(C)$ be the universal odd derivation over $k$ acting on generators of $C$ as $d\left(\mathbf{k} u_{i, \alpha \beta}^{(m)}\right)=(-1)^{p(\mathbf{k})}$ $\mathrm{k} d u_{i, \alpha \beta}^{(m)}, \mathrm{k} \in k$; thus, $p\left(d u_{i, \alpha \beta}^{(m)}\right)=1+p\left(u_{i, \alpha \beta}^{(m)}\right)=1+p(\alpha)+$ $p(\beta)$. We uniquely extend $\partial$ on $\Omega^{1}(C)$ by requiring it to commute with $d$. Set $C^{\prime}=C\left(\left(\xi^{-1}\right)\right):=\left\{c^{\prime}=\Sigma_{r=-\infty}^{N} c_{r} \xi^{r} \mid N<\infty\right.$, $\left.c_{r} \in C\right\}, \Omega^{\prime}=\Omega\left(\left(\xi^{-1}\right)\right)$, with the associative multiplication in $C^{\prime}, C$-actions on $C^{\prime}$, and $C^{\prime}$-actions on $\Omega^{\prime}$ made possible by the relations $\xi^{m} f=\sum_{r=0}^{m}\left(\begin{array}{c}m \\ r\end{array}\right)\left(\partial^{r} f\right) \xi^{m-r}, \xi^{-m-1} f=\Sigma_{r=0}^{\infty}$ $(-1)^{r}\left(\begin{array}{c}m+\eta \\ r\end{array}\left(\partial^{r} f\right) \xi^{-m-r-1}, m \geq 0, f \in C\right.$ or $\Omega$ (see ref. 11). The $p$-grading on $C^{\prime}$ and $\Omega^{\prime}$ is defined as $p\left(f \xi^{\prime}\right)=p(f), f \in C$ or $\Omega$. The derivations $d$ and $\partial$ are naturally extended on $C^{\prime}, \bigcirc_{C}$, and $O_{C^{\prime}}$ while $\partial$ also acts on $O_{\Omega}$ and $O_{\Omega^{\prime}}$ (see ref. 11). We write $a \sim b$ if $(a-b) \in \operatorname{Im} \partial$.

We introduce $w$-grading on $C, C^{\prime}, \Omega, \Omega^{\prime}, \bigcirc_{C^{\prime}}$, and $\bigcirc_{\Omega^{\prime}}$ by $w\left(u_{i, \alpha \beta}^{(m)}\right)=w\left(d u_{i, \alpha \beta}^{(m)}\right)=n-i+m, w(\xi)=1, w(k)=0$, and $w(X)=w\left(X_{\alpha \beta}\right)$ for $X \in \bigcirc_{V}, X_{\alpha \beta} \in V$. For $Q=\Sigma q_{r} \xi^{r} \in C^{\prime}$ or $\bigcirc_{C^{\prime}}$ or $\Omega^{\prime}$ or $\bigcirc_{\Omega^{\prime}}$, we define $Q_{+}=\Sigma_{r \geq 0} q_{r} \xi^{r}, Q_{-}=Q-Q_{+}$, and $\operatorname{Res} Q=q_{-1}$.

A derivation $X$ of $C$ over $k$ is called evolutionary if it commutes with $\partial$. Such an evolution derivation is uniquely defined by its action on the generators $u_{i, \alpha \beta}: X=\Sigma\left[\partial^{m}(X\right.$ $\left.\left.\left(u_{i, \alpha \beta}\right)\right)\right] \cdot \partial / \partial u_{i, \alpha \beta}^{(m)}$, where $\partial / \partial u_{i, \alpha \beta}^{(m)}$ is the natural derivation of $C$ over $k$ of parity $p(i)$. The Lie superalgebra of evolution derivations is denoted $D^{e v}(C)$; the $\mathbf{Z}_{2}$-grading on $D^{e v}(C)$ is given by $p(X)=p\left(X\left(u_{i, \alpha \beta}\right)\right)+p(\alpha)+p(\beta), X \in D^{e v}(C)$.

2. The Centralizer. We set $L=\Sigma_{i=0}^{n} u_{i} \xi^{i} \in \bigcirc_{C^{\prime}}$, where $u_{i}$ $=\left(u_{i, \alpha \beta}\right)$ are matrices satisfying the following conditions: $(i)$ $u_{n}$ is even, $\partial$-constant (i.e., $u_{n} \in O_{k}$ ), invertible, and diagonalizable, which we shall consider already diagonal: $u_{n}=$ $\operatorname{diag}\left(\mathrm{k}_{1}, \ldots, \mathrm{k}_{\ell}\right), \mathrm{k}_{\alpha} \in k_{0}$. Let $\pi: k \rightarrow k_{0} /\left(k_{1}\right)$ denote the natural homomorphism (see ref. 13). We require that if $\pi\left(\mathbf{k}_{\alpha}\right)=$ $\pi\left(k_{\beta}\right)$ then $k_{\alpha}=k_{\beta}$ [i.e., the dimension of the center of the centralizer is the same for $u_{n}$ and $\left.\pi\left(u_{n}\right)\right]$. (ii) $u_{n-1} \in \operatorname{Im}$ ad $u_{n}$ in $\left(O_{C}\right)^{L i e}$-i.e., $u_{n-1, \alpha \beta}=0$ when $k_{\alpha}=k_{\beta}$. 
Accordingly, we specify $C=k\left[u_{i, \alpha \beta}^{(m)}\right]$ such that $0 \leq i \leq n-$ 1 and $u_{n-1, \alpha \beta}$ is absent whenever $\mathbf{k}_{\alpha}=\mathbf{k}_{\beta}$.

Denote $Z(L)$ the centralizer of $L$ in $\bigcirc_{C^{\prime}}$. Let $Q \in Z(L), Q$ $=\Sigma^{a} q_{r} \xi^{r}$, and suppose $Q$ is $p$-homogeneous. Comparing the $\xi^{n+a-x}$-coefficients in the equality $Q L=L Q$, for $x=0,1,2$, we find that $q_{a}$ is $\partial$-constant, diagonal, and $(-1)^{p(\alpha) p\left(q_{a}\right)} q_{a, \alpha \alpha}$ $=(-1)^{p(\beta) p\left(q_{a}\right)} q_{a, \beta \beta}$ whenever $\mathrm{k}_{\alpha}=\mathrm{k}_{\beta}$. The elements $q_{a} \xi^{a}$ with such $q_{a}$ are called admissible.

THEOREM 1. Let $\mathrm{q}_{\mathrm{a}} \xi^{\mathrm{a}}$ be an admissible $\mathrm{p}$-homogeneous element. Then (i) $\mathrm{Z}(\mathrm{L})$ contains unique $\mathrm{p}$-homogeneous element of the form $\mathrm{Q}=\mathrm{q}_{\mathrm{a}} \xi^{\mathrm{a}}+$ (lower order in $\xi$ terms) which is $\mathrm{w}$-homogeneous of degree a; (ii) $\mathrm{Z}(\mathrm{L})$, as $\mathrm{k}$-bimodule, consist precisely of the sums of such elements; (iii) $(\mathrm{Z}(\mathrm{L}))^{\mathrm{Lie}}$ is trivial, that is, any two elements in $\mathrm{O}_{\mathrm{C}^{\prime}}$ that commute with $\mathrm{L}$ supercommute between themselves.

3. The Equations. For each $w$ - and $p$-homogeneous $Q \in$ $Z(L)$ we define an evolution derivation $\partial_{Q}$ in $C$ by

$$
\partial_{Q}(L)=\left[Q_{+}, L\right]=\left[-Q_{-}, L\right],
$$

with the bracket $[$,$] taken in \left(\bigcirc_{C^{\prime}}\right)^{\text {Lie }}$. Thus, $\partial_{Q}\left(u_{n-1}\right) \in$ Im ad $u_{n}$, and $p\left(\partial_{Q}\right)=p(Q)$. Eqs. 1 are of the Lax type.

THEOREM 2. For $\mathrm{Q}, \mathrm{P} \in \mathrm{Z}(\mathrm{L})$, (i) $\left[\partial_{\mathrm{Q}}, \partial_{\mathrm{P}}\right]=0$, i.e. $\partial_{\mathrm{P}}$ and $\partial_{\mathrm{Q}}$ supercommute; (ii)

$$
\partial_{Q}(P)=\left[Q_{+}, P\right]=\left[-Q_{-}, P\right] .
$$

THEOREM 3. Let $\overline{\mathrm{C}} \supset \mathrm{C}$ be a $\mathbf{Z}_{2}$-graded extension of $\mathrm{C}$, with a w-grading on $\overline{\mathrm{C}}$ agreeing with that of $\mathrm{C}$, with a derivation $\bar{\partial}: \bar{C} \rightarrow \bar{C}$ being even, surjective, agreeing with $\partial$ on $\mathrm{C}$, having only elements of $\mathrm{k}$ in its kernel, and having $\mathrm{w}$-weight one (see ref. I). (i) Then there exists and unique an element $\mathrm{K} \in \mathrm{O}_{\overline{\mathrm{C}}^{\prime}}, \mathrm{w}(\mathrm{K})=0, \mathrm{p}(\mathrm{K})=0$, of the form $\mathrm{K}=1+\Sigma_{\mathrm{r}>0}$ $\chi_{\mathrm{r}} \xi^{-\mathrm{r}}, \chi_{\mathrm{r}} \in \mathrm{O}_{\overline{\mathrm{C}}}$, such that $\mathrm{K}^{-1} \mathrm{LK}=\mathrm{u}_{\mathrm{n}} \xi^{\mathrm{n}}$. (ii) Let $\partial_{\mathrm{Q}}$ be uniquely extended on $\overline{\mathrm{C}}$ by requiring it to commute with $\bar{\partial}$ and being $\mathrm{w}$ - and $\mathrm{p}$-homogeneous. Then $\partial_{\mathrm{Q}}(\mathrm{K})=-\mathrm{Q}_{-} \mathrm{K}$.

Remarks: (i) From Theorem 1 it follows that we can restrict ourselves only to even elements $Q$ of $Z(L)$ by requiring $q_{a}$ to have only ones and zeroes on the diagonal. (ii) To avoid the introduction of the extension $\bar{C} \supset C$, one could start directly with $K$, define $\bar{C}$ as $k\left[\chi_{r, \alpha \beta}^{(m)}\right]$ and $L$ as $K u_{n} \xi^{n} K^{-1}$, and thereafter define $C$ as $k\left[u_{i, \alpha \beta}^{(m)}\right]$. Then Theorem 3 amounts to the statement that $K q_{a} \xi^{a} K^{-1} \in \bigcirc_{C^{\prime}}$ for admissible elements $q_{a} \xi^{a}$. This point of view was suggested for the differential Lax equations in ref. 14 and was taken for the discrete Lax equations in chapter IX of ref. 15.

4. The Conservation Laws and Their Variational Derivatives. For $q \in O_{C}$ we define the supertrace str $q=\Sigma_{\alpha}$ $(-1)^{p(\alpha)[1+p(q)]} q_{\alpha \alpha}$. Recall that $\operatorname{str}\left(\left[q, q^{\prime}\right]\right)=0$ for $q, q^{\prime} \in$ $\left(\mathrm{O}_{C}\right)^{\text {Lie }}$ (ref. 13).

LEMMA 1. Define a k-bilinear form on $\bigcirc_{C^{\prime}}\left(\right.$ or $\left.\left(\bigcirc_{C^{\prime}}\right)^{\text {Lie }}\right)$ by

$$
(\mathrm{X}, \mathrm{Y})=\operatorname{sir} \operatorname{Res}(\mathrm{XY}) \text {. }
$$

Then (i) $(\mathrm{X}, \mathrm{Y}) \sim(\mathrm{Y}, \mathrm{X})(-1)^{\mathrm{p}(\mathrm{X}) \mathrm{p}(\mathrm{Y})}$. Equivalently, str Res $([X, Y]) \sim 0$. (ii) The form (. , .) is invariant (modulo Im d) on $\left(\mathrm{O}_{\mathrm{C}^{\prime}}\right)^{\mathrm{Lie}}:(\mathrm{X},[\mathrm{Y}, \mathrm{Z}]) \sim([\mathrm{X}, \mathrm{Y}], \mathrm{Z}), \mathrm{X}, \mathrm{Y}, \mathrm{Z} \in\left(\mathrm{O}_{\mathrm{C}^{\prime}}\right)^{\mathrm{Lie}}$.

THEOREM 4. (i) Each super-Lax Eq. 1 has an infinite common set of conservation laws str Res $(\mathrm{Q}), \mathrm{Q} \in \mathrm{Z}(\mathrm{L})$, $\mathrm{w}(\mathrm{Q}) \geq 0$. (ii) Let $\Theta$ be the dimension of the center of the centralizer of $u_{n}$ in $O_{k}(=$ the number of different elements amongst $\left.k_{1}, \ldots, k_{\ell}\right)$. Then, for each nonnegative integer a not divisible by $\mathrm{w}(\mathrm{L})$, there exists $\Theta$ linearly independent over $\mathrm{k}$ even conservation laws of the Eq. 1 with $\mathrm{w}$-weight $\mathrm{a}+1$, coming from $\Theta$ linearly independent, even, admissible elements $\mathrm{q}_{\mathrm{a}} \xi^{\mathrm{a}}$; for $\mathrm{a}: \mathrm{n}$, the corresponding number is $\Theta-1$.

For $w$ - and $p$-homogeneous $Q \in Z(L)$, with $w(Q)>0$, denote $H_{Q}=[n / w(Q)]$ str $\operatorname{Res}(Q)$.

LemMa 2. For $\mathrm{w}$ - and p-homogeneous $\mathrm{Q}, \mathrm{P} \in \mathrm{Z}(\mathrm{L})$,

$$
\begin{gathered}
\mathrm{w}(\mathrm{P}) \operatorname{str} \operatorname{Res}(\mathrm{PdQ}) \\
\sim(-1)^{\mathrm{p}(\mathrm{P})+\mathrm{p}(\mathrm{Q})+\mathrm{p}(\mathrm{P}) \mathrm{p}(\mathrm{Q})} \mathrm{w}(\mathrm{Q}) \operatorname{str} \operatorname{Res}(\mathrm{QdP}) .
\end{gathered}
$$

Corollary 1.

$$
\mathrm{dH}_{\mathrm{LQ}} \sim \operatorname{str} \operatorname{Res}(\mathrm{dL} \circ \mathrm{Q})
$$
2 .

Set $Q=\Sigma \xi^{r} q_{r}$. Writing 5 in longhand, we obtain Corollary

COROllary 2.

$$
\mathrm{q}_{-\mathrm{i}-1, \alpha \beta}=(-1)^{\mathrm{p}(\beta) \mathrm{p}(\mathrm{Q})} \frac{\delta \mathrm{H}_{\mathrm{LQ}}}{\delta \mathrm{u}_{\mathrm{i}, \beta \alpha}}, 0 \leq \mathrm{i} \leq \mathrm{n}-1,
$$

where $\delta / \delta \mathrm{u}_{\mathrm{i}, \alpha \beta}=\Sigma_{\mathrm{m}}(-\partial)^{\mathrm{m}} \partial / \partial \mathrm{u}_{\mathrm{i}, \alpha \beta}^{(\mathrm{m})}$ and in Eq. 6 only those terms corresponding to $\mathrm{i}=\mathrm{n}-1$ are present for which $k_{\alpha} \neq$ $k_{\beta}$. form

Thus, for an even $Q \in Z(L)$, one can rewrite Eq. 6 in the

$$
\begin{aligned}
Q_{-}= & \xi^{-1} \frac{\delta H}{\delta u_{0}^{t}}+\xi^{-2} \frac{\delta H}{\delta u_{1}^{t}}+\cdots \\
& +\xi^{-n} \frac{\delta H}{\delta u_{n-1}^{t}}+\cdots, H=H_{L Q} .
\end{aligned}
$$

Substituting Eq. 6 into Eq. 1, we arrive at an expression of the form $\partial_{Q}\left(u_{i, \alpha \beta}\right)=(-1)^{p(H)(p(\alpha)+p(\beta)]} \Sigma_{j, \mu \nu} B_{i, \alpha \beta l j, \mu \nu}$ $\left(\delta H / \delta u_{j, \mu \nu}\right), H=H_{L Q}$, with some matrix $B \in \bigcirc_{C^{\prime}}$. In contrast to the commutative case (12), the matrix $B$ is not superHamiltonian. This loss of the first Hamiltonian structure makes a really significant difference between general superand usual integrable systems.

This work was supported in part by the National Science Foundation and the Department of Energy.

1. Wilson, G. (1979) Math. Proc. Cambridge Philos. Soc. 86, 131-143.

2. Drinfel'd, V. G. \& Sokolov, V. V. (1981) Sov. Math. Dokl. 23, 457-462.

3. Wilson, G. (1981) Ergod. Theory Dyn. Systems 1, 361-380.

4. Dresden, M. \& Chen, S. F. (1976) Physica A 83, 1-17.

5. Corwin, L., Ne'eman, Y. \& Sternberg, S. (1975) Rev. Mod. Phys. 47, 573-603.

6. Deser, S. (1981) Hadronic J. 4, 19-46.

7. D'Auria, R. \& Sciuto, S. (1980) Nucl. Phys. B 171, 189-208.

8. Olshanetsky, M. A. (1983) Commun. Math. Phys. 88, 63-76.

9. Kac, V. G. (1977) Adv. Math. 26, 8-96.

10. Kupershmidt, B. A. \& Wilson, G. (1981) Commun. Math. Phys. 81, 189-202.

11. Manin, Yu. I. (1979) J. Sov. Math. 11, 1-122.

12. Kupershmidt, B. A. \& Wilson, G. (1981) Invent. Math. 62, $403-436$.

13. Leites, D. A. (1980) Russ. Math. Surv. 35, 1-64.

14. Wilson, G. (1981) Q. J. Math. Oxford (2nd Ser.) 32, 491-512.

15. Kupershmidt, B. A. (1982) Discrete Lax Equations and Differential-Difference Calculus, Ecole Norm. Super. Lecture Notes (Paris). 